\title{
Brouwers Fixed Point Theorem
}

\author{
Yogesh Chandra C \\ Senior secondary NIOS Bangalore, 93/5 ,8th Cross, Maruthi Road, Shakambarinagar, Bangalore, India -560078
}

\begin{abstract}
The theorem is important illustration of progress of branch algebraic topology and base for functional analysis and fixed point theorem. Provides generalization and proof to fixed point theorem, game theory, central limit theorem and gave way to new branch of mathematics Fixed point theory used extensively. Lack of interest in analysis situ, unnoticed theory, use of false intuitionism against set theory created problems, the method used is continuous mapping, Euclidean spaces, algebraic topology, the results of this theory are unconstructive and let to constructivity idea. Thus theory provided significant proof, generalization to many important theorem.
\end{abstract}

Keywords: Homology; Euclidean space; Banach space; one dimensional scale; topology; contraction; vector fields

\section{Structure}

This theorem comes from a branch of math known as Topology, and was discovered by LuitzenBrouwer. While its technical expression is quite abstract, it has many fascinating real world implications. Let's say we have a picture (for example, the Mona Lisa) and we take a copy of it. We can then do whatever we want to this copy-make it bigger, make it smaller, rotate it, crumple it up, anything. Brouwer's Fixed Point Theorem says that if we put this copy overtop of our original picture, there has to be at least one point on the copy that is exactly overtop the same point on the original. It could be part of Mona's eye, ear, or possible smile, but it has to exist.

This also works in three dimensions: imagine we have a glass of water, and we take a spoon and stir it up as much as we want. By Brouwer's theorem, there will be at least one water molecule that is in the exact same place as it was before we started stirring.

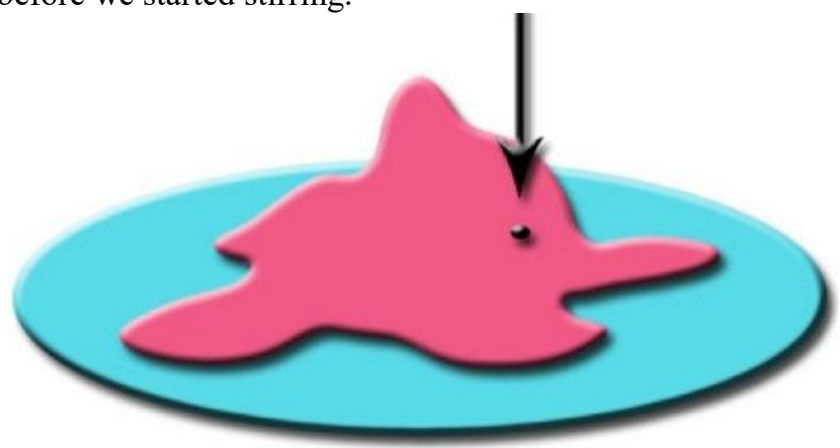

Figure 1: Representative Picture

\section{Discussions}

The theorem provides certain statements

The theorem has several formulations, depending on the context in which it is used and its degree of generalization. The simplest is sometimes given as follows:

In the plane:

- Every continuous function from a closed disk to itself has at least one fixed point. ${ }^{[6]}$

This can be generalized to an arbitrary finite dimension:

In Euclidean space:

- Every continuous function from a closed ball of a Euclidean space into itself has a fixed point. ${ }^{[7]}$

A slightly more general version is as follows:[

Convex compact set:
- Every continuous function from a convex compact subset $K$ of a Euclidean space to $K$ itself has a fixed point. An even more general form is better known under a different name:

Schauder fixed point theorem:

- Every continuous function from a convex compact subset $K$ of a Banachspace to $K$ itself has a fixed point.

\section{Experiment}

Suppose that $\mathrm{S}$ is a set. A function $\mathrm{f}: \mathrm{S} \rightarrow \mathrm{S}$ has a fixed point if there is an element $x \in S$ so that $f(x)=x$. A fixed point theorem is a theorem like this: with some conditions on $\mathrm{S}$ or $\mathrm{f}$ or both, f must have a fixed point.

\section{EXAMPLES}

Any contraction from $\mathrm{R}$ to $\mathrm{R}$ has a fixed point.

The intermediate value theorem implies that every continuous function $\mathrm{f}:[0,1] \rightarrow[0,1]$ has a fixed point. A function $\mathrm{f}:[0,1] \rightarrow[0,1]$ is a contraction if $\mathrm{f}$ contracts distances:

for all $\mathrm{x} 1, \mathrm{x} 2 \in[0,1],|\mathrm{x} 1-\mathrm{x} 2|>|\mathrm{f}(\mathrm{x} 1)-\mathrm{f}(\mathrm{x} 2)|$.

\section{Proof}

A function $\mathrm{f}:[0,1] \rightarrow[0,1]$ is a contraction if $\mathrm{f}$ contracts distances:

for all $\mathrm{x} 1, \mathrm{x} 2 \in[0,1],|\mathrm{x} 1-\mathrm{x} 2|>|\mathrm{f}(\mathrm{x} 1)-\mathrm{f}(\mathrm{x} 2)|$.

Theorem: Any contraction $\mathrm{f}:[0,1] \rightarrow[0,1]$ has a unique fixed point.

Outline of proof: Pick any $x 0 \in[0,1]$. Define a sequence $\{\mathrm{x} 0, \mathrm{x} 1, \mathrm{x} 2, \ldots\}$

By $\mathrm{x} 1=\mathrm{f}(\mathrm{x} 0)$

$x 2=f(x 1)=f(f(x 0))$

$x 3=f(x 2)=f(f(f(x 0)))$

$\ldots$ Then $\lim n \rightarrow \infty x$ is the (unique) fixed point of $f$

\section{Illustrations}

1) Take two sheets of graph paper of equal size with coordinate systems on them, lay one flat on the table and crumple up (without ripping or tearing) the other one and place it, in any fashion, on top of the first so that the crumpled paper does not reach outside the flat one. There will then be at least one point of the crumpled sheet that lies directly above its corresponding point (i.e. the point 


\section{International Journal of Science and Research (IJSR) \\ ISSN (Online): 2319-7064}

Index Copernicus Value (2013): 6.14 | Impact Factor (2014): 5.611

with the same coordinates) of the flat sheet. This is a consequence of the $n=2$ case of Brouwer's theorem applied to the continuous map that assigns to the coordinates of every point of the crumpled sheet the coordinates of the point of the flat sheet immediately beneath it.

2) Take an ordinary map of a country, and suppose that that map is laid out on a table inside that country. There will always be a "You are Here" point on the map which represents that same point in the country.

3) In three dimensions the consequence of the Brouwer fixed-point theorem is that, no matter how much you stir a cocktail in a glass, when the liquid has come to rest some point in the liquid will end up in exactly the same place in the glass as before you took any action, assuming that the final position of each point is a continuous function of its original position, and that the liquid after stirring is contained within the space originally taken up by it

\section{One Dimension Scale}

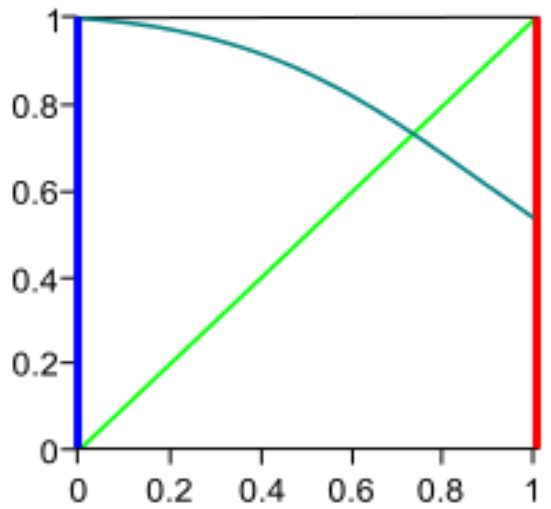

Figure 2: one dimensional scale

In one dimension, the result is intuitive and easy to prove. The continuous function $f$ is defined on a closed interval $[a$, $b]$ and takes values in the same interval. Saying that this function has a fixed point amounts to saying that its graph (dark green in the figure on the right) intersects that of the function defined on the same interval $[a, b]$ which maps $x$ to $x$ (light green).

Intuitively, any continuous line from the left edge of the square to the right edge must necessarily intersect the green diagonal. Proof: consider the function $g$ which maps $x$ to $f(x)$ - $x$. It is $\geq 0$ on $a$ and $\leq 0$ on $b$. By the intermediate value theorem, $g$ has a zero in $[a, b]$; this zero is a fixed point.

Brouwer is said to have expressed this as follows: "Instead of examining a surface, we will prove the theorem about a piece of string. Let us begin with the string in an unfolded state, then refold it. Let us flatten the refolded string. Again a point of the string has not changed its position with respect to its original position on the unfolded string."

\section{Conclusion}

\section{Using Homology}

The proof uses the observation that the boundary of $D^{n}$ is $S^{n-1}$, the $(n-1)$-sphere.

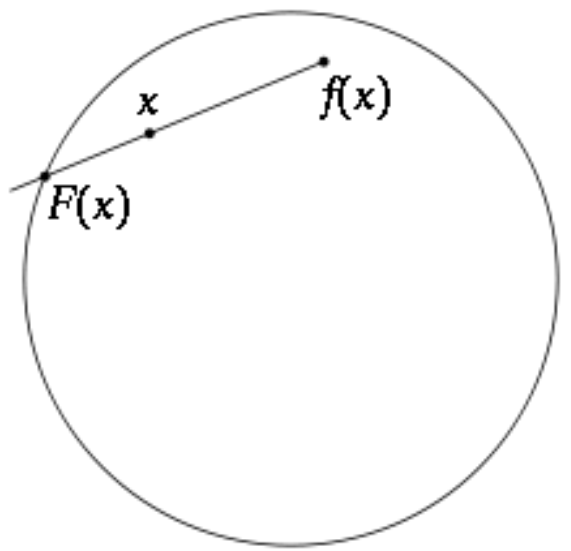

Figure 3: Retraction $F$

The argument proceeds by contradiction, supposing that a continuous function $f: D^{n} \rightarrow D^{n}$ has no fixed point, and then attempting to derive an inconsistency, which proves that the function must in fact have a fixed point. For each $x$ in $D^{n}$, there is only one straight line that passes through $f(x)$ and $x$, because it must be the case that $f(x)$ and $x$ are distinct by hypothesis (recall that $f$ having no fixed points means that $f(x) \neq x)$. Following this line from $f(x)$ through $x$ leads to a point on $S^{n-1}$, denoted by $F(x)$. This defines a continuous function $F: D^{n} \rightarrow S^{n-1}$, which is a special type of continuous function known as a retraction: every point of the codomain (in this case $S^{n-1}$ ) is a fixed point of the function.

Intuitively it seems unlikely that there could be a retraction of $D^{n}$ onto $S^{n-1}$, and in the case $n=1$ it is obviously impossible because $S^{0}$ (i.e., the endpoints of the closed interval $D^{1}$ ) is not even connected. The case $n=2$ is less obvious, but can be proven by using basic arguments involving the fundamental groups of the respective spaces: the retraction would induce an injective group homomorphism from the fundamental group of $S^{1}$ to that of $D^{2}$, but the first group is isomorphic to $\mathrm{Z}$ while the latter group is trivial, so this is impossible. The case $n=2$ can also be proven by contradiction based on a theorem about nonvanishing vector fields.

For $n>2$, however, proving the impossibility of the retraction is more difficult. One way is to make use of homology groups: the homology $H_{n-1}\left(D^{n}\right)$ is trivial, while $H_{n-1}\left(S^{n-1}\right)$ is infinite cyclic. This shows that the retraction is impossible, because again the retraction would induce an injective group homomorphism from the latter to the former group.

\section{References}

[1] "Brouwer's fixed point theorem, perhaps the most important fixed point theorem." p xiii V. I. Istratescu Fixed Point Theory an Introduction Kluwer Academic Publishers (new edition 2001) ISBN 1-4020-0301-3.

[2] S. Greenwood J. Cao Brouwer's Fixed Point Theorem and the Jordan Curve Theorem University of Auckland, New Zealand.

[3] Schauder, J. (1930). "Der Fixpunktsatz in Funktionsräumen". Studia. Math. 2: 171-180.

[4] D. Violette Applications du lemme de Sperner pour les triangles Bulletin AMQ, V. XLVI N ${ }^{\circ} 4$, (2006) p17.

\section{Volume 4 Issue 11, November 2015}




\section{International Journal of Science and Research (IJSR) \\ ISSN (Online): 2319-7064}

Index Copernicus Value (2013): 6.14 | Impact Factor (2014): 5.611

Page 15 of: D. Leborgne Calculdifférentieletgéométrie Puf (1982) ISBN 2-13-037495-6.

[5] This version follows directly from the previous one because every convex compact subset of a Euclidean space ishomeomorphic to a closed ball of the same dimension as the subset; see Florenzano, Monique (2003).

[6] General Equilibrium Analysis: Existence and Optimality Properties of Equilibria. Springer.p. 7. ISBN 9781402075124.

[7] V. \& F. Bayart Point fixe, etthéorèmes du point fixe on Bibmath.net.

[8] C. Minazzo K. Rider Théorèmes du Point Fixe et Applications aux Equations Différentielles Université de Nice-Sophia Antipolis. 